\section{LA AUTOAYUDA Y EL CONSUMO DE DISPOSITIVOS PSÍQUICOS EN EL CASO DE LA INNOVACIÓN}

\author{
Eduardo Apodaka \\ Universidad del País Vasco-Euskal Herriko Unibertsitatea \\ Departamento de Psicología Social \\ eduardo.apodaka@ehu.es
}

\section{SELF-HELP AND THE CONSUME OF PSYCHIC DISPOSITIVES IN HE CASE OF INNOVATION}

\begin{abstract}
Innovation appears in the business world and, generally, in the organizational spheres, as a demand of the economic system that is characteristic of informational capitalism and its' predominant logics: the search of and added value. This is done parallel to the cultural logics that have cleaned out and taken down the modern psychic individual. The initiative and the motivation have passed from being a raw material to a high-elaborated product, a product that can be worked apart form the individual through the traditional ways or be included in an psychoanalyzed auto construction. This text analyses the psycho-cultural function and the success of the selfhelp books along with its' "psycho-ideology." The culture of self-help has as a horizon all types of relational spheres and all dispositions, feelings, and emotions. This article is focused on the case of innovation, that is, the analysis of the functions of the books of self-help that are directed to make people more innovative and proactive.
\end{abstract}

KEY WORDS: Innovation; new management; self-help; modern psychism; added value.

\begin{abstract}
RESUMEN: La innovación aparece en el mundo de la empresa y, en general, en los ámbitos organizacionales, como demanda del sistema económico propio del capitalismo informacional y de su lógica imperante: la búsqueda del valor añadido. Y lo hace en concurso con lógicas culturales que han ido vaciando y desmontando al sujeto psíquico moderno. La iniciativa y la motivación han pasado de materia prima a producto altamente elaborado. Un producto que puede ser trabajado desde fuera del sujeto por las vías tradicionales, o bien inserto en una autoconstrucción psicologizante. Este texto analiza la función psicocultural y el éxito de los libros de autoayuda junto con su "psico-ideología". Si bien la cultura de la autoayuda tiene como horizonte todo tipo de ámbito de relación y todas las disposiciones, sentimientos y emociones, el artículo se ocupa del caso de la innovación, es decir, del análisis de la función de los libros de autoayuda destinados a convertir a la gente en innovadora y proactiva.
\end{abstract}

PALABRAS CLAVE: Innovación; nuevo management; autoayuda; psiquismo moderno; valor añadido.

The choices, after all, are yours. You choose happiness. You choose sadness. You choose decisiveness. You choose ambivalence. You choose success. You choose failure. You choose courage. You choose fear (...) Be Proactive is about taking responsibility for your life...

Stephen CoveY

The Seven Habits of Highly Effective PeopleYes, we can! David Axelrod-Barack Obama

\section{INTRODUCCIÓN: LA AUTOAYUDA Y EL DISCURSO DEL NUEVO MANAGEMENT ${ }^{1}$}

La primera dificultad en el análisis de la autoayuda es su indefinición o su múltiple, variada y compleja omnipresencia. Si nos remitimos a los libros de autoayuda podemos definirlos como libros "prácticos" destinados a asesorar al lector para afrontar alguna consecuencia perniciosa de la cultura o de la sociedad actual. En la mayoría de los casos van más lejos y pretenden tener la solución a los problemas personales y las claves para alcanzar una vida mejor (Dolby 2005,38 ). Ahora bien, los libros no son más que un soporte. Y la autoayuda se extiende mucho más allá. La lista de las expresiones de la autoayuda es larga: la autoayuda es imprecisa y multiforme. Junto con los libros encontramos programas de televisión (los talk shows, las super-nannys 
y todo tipo de programa "consejero"), películas y documentales empapados de autoayuda, revistas especializadas o secciones en revistas no especializadas, foros, blogs, páginas web y un sinfín de recursos de autoayuda en Internet; disponemos además de una tupida red de terapeutas y consejeros, algunos titulados y colegiados, otros no, y existen numerosas agrupaciones de autoayuda. $Y$, como colofón, aumentan día a día las numerosas aportaciones de la autoayuda naturalizadas en el lenguaje cotidiano.

La autoayuda es, por tanto, un complejo con expresiones muy diferentes que exigen análisis particulares, pero en este artículo pretendo contextualizar la autoayuda, su discurso y sus prácticas, en un marco más extenso que explique las transformaciones de la psique moderna, el desarrollo de sus modelos ideales y su declive. El objetivo último del artículo es situar en ese marco el discurso del denominado Nuevo Management y en particular uno de sus ejes centrales, el tópico de la innovación. El Nuevo Management se ha destacado por ser un buen receptor de las propuestas de las disciplinas psicológicas y de la autoayuda, copiando y emulando sus discursos, sus prácticas y sus técnicas en una particular "ingenieria psicosocial" aplicada al mundo organizacional y laboral. Como voy a intentar mostrar, las demandas del capitalismo actual y de sus actores y las derivas socioculturales se expresan y tienen respuesta en el complejo de la autoayuda en todas sus versiones, especialmente en el mundo organizacional.

Los estudios sobre la autoayuda se pueden clasificar según dos aproximaciones generales: la retórica-discursiva y la pragmática. En la primera encontramos abordajes foucaultianos que siguiendo la estela de los estudios de Nikolas Rose $(1990,1996)$ consideran la autoayuda como un nuevo discurso disciplinario y pretenden desvelar lo que oculta. En la segunda encontramos aquellos que como Eva Illouz piensan que la sospecha debe dejar lugar a una crítica inmanente que partiendo de los textos y las prácticas se ocupe de lo que la gente hace con ellos.

La primera, por tanto, consiste en prestar atención al contenido discursivo de las expresiones de la autoayuda para ir más allá. En esa perspectiva, el discurso de la autoayuda denuncia que nuestra cultura (el "sistema sociocultural" por sintetizar lo que en los libros de autoayuda se menciona de infinitas maneras, pero aparece siempre como horizonte contra el cual se confronta la persona) produce graves sufrimientos psicológicos o personales, dichos sufrimientos se pueden y se deben afrontar y superar despertando en uno mismo energías psíquicas latentes. La persona debe saber que es un motor que funciona a base de energía psíquica endógena. Pero lo que el discurso oculta y produce es la psicologización esencialista del self y, por ende, una hiper-individualización (Rimkie, 2000, 62) o sobre-elaboración del yo (McGee, 2005). El discurso de la autoayuda psicológica tiene, según esta perspectiva, un papel clave en las "democracias liberales avanzadas" contribuyendo a la gobernabilidad de los ciudadanos, puesto que establece por un lado el modelo normativo de persona sana (gobernable, calculable, clasificable, consciente, responsable, autorregulado y autodeterminado, Rose, 1990, 1996) y, por otro, la creencia en que cada persona posee un centro de agencia que la capacita para actuar a voluntad sobre sí misma y en cierta medida sobre los demás. Como señala Rimkie $(2000,65)$ la lección moral es sencilla: los individuos "pobres de espíritu" no son más que individuos que han dejado de gobernarse a sí mimos.

La segunda aproximación, la pragmática, pretende entender el uso de la autoayuda sin presuponer un modelo correcto contra el que criticar la autoayuda. Eva Illouz lo plantea así: "los significados y las ideas deberían ser vistos como herramientas útiles, esto es, como herramientas que nos permiten llevar a cabo ciertas cosas en la vida diaria" $(2010,16)$. Según esta autora la autoayuda hunde sus raíces en una alianza entre representaciones del yo y el discurso especializado de la terapia. Su éxito es producto de su utilidad: un fondo cultural (la creencia en el yo en los Estados Unidos de América) propició la descarga "pragmática" de un discurso especializado, la psicoterapia (Philip, 1966). La terapia llegó de ese modo "a constituirse como uno de los principales códigos con los cuales expresar, conformar y guiar el yo" (IIlouz, 2010, 17). Así que el éxito de la autoayuda se debe a que da sentido a la experiencia social de quienes la usan, proporciona guias prácticas para hacer frente a "áreas cargadas de conflictividad de la conducta social" (IIlouz, 2010, 35) y, sobre todo, a que ha sido previamente institucionalizada como marco de la experiencia posible del yo (Moskowitz, 2001).

Una aproximación pragmática "compasiva" con las personas "usuarias" de la autoayuda intenta ver en los usos formas positivas de despliegue personal, procura apreciar los usos divergentes, las formas de creatividad y autonomía 
espontánea que puedan darse en esas prácticas, etc. $Y$, en definitiva, toma en consideración las bondades directas 0 indirectas, intencionadas o no, de la autoayuda. No obstante, una aproximación pragmática menos integrada en la naturalidad de la práctica se fija no tanto en lo que la gente hace como en lo que el complejo de la autoayuda hace con la gente $y$, sobretodo, en el tipo de gente que hace. Por esa vía, la actitud pragmática se encuentra de inmediato con la crítica retórico-discursiva en torno a la cuestión central de la autoayuda: la presunción de que existe un núcleo psíquico individual oprimido y paralizado por la presión externa "sociocultural". Pese a las resistencias, divergencias y modificaciones creativas que puedan moderar e incluso modificar esa presunción básica, el complejo funciona en sus términos expresos porque concurre con cambios socioculturales "macro": individualización, desocialización, desincronización social, erosión de lo común, etc. Esos cambios explican por qué funciona la autoayuda y en cierta manera cómo funciona en la extensión masiva de la elaboración hipertrófica del yo, en la habilitación de los sujetos y en el auxilio paliativo de los males que acompañan a esos procesos.

\section{El COMPLEJO DE AUTOAYUdA}

A la hora de caracterizar la autoayuda Steve Salerno (2005) habla de "movimiento" (SHAM o Self-Help American Movement). Un movimiento que hunde sus raices en la cultura americana desde tiempos coloniales (le atribuye a Benjamín Franklin el primer libro de autoayuda: Poor Richard's Almanack, 1732) pero que adquirió su forma actual en 1967 con la publicación del best seller de Thomas A. Harris I'm OK-You're OK. Entonces, dice Salerno, apareció el gurú y el simple consejero se convirtió en un adivino motivacional (motivational soothsayer). $Y$ es cierto que en Estados Unidos la autoayuda tiene características que la acercan a un movimiento social, aunque dada su amplitud y su profunda relación con las matrices culturales de los norteamericanos quizás sería más correcto hablar de la autoayuda como un vasto movimiento cultural (Eva Illouz habla de "cultura de la autoayuda", 2007, 2010).

Por otro lado, Ingleby (1985) y Rose $(1985,1990,1996)$ han hablado del "psy complex", esto es, del conjunto de disciplinas, discursos, prácticas y técnicas psicoterapéuti- cas. En principio este complejo está formado por las disciplinas "psico-expertas" (psicología, psiquiatría, psicoanálisis) pero ha sobredeterminado el discurso y las prácticas de la enfermería y la asistencia sanitaria, la mediación y el trabajo social, el asesoramiento laboral, profesional o académico, la gestión empresarial, la política... Y la autoayuda, que en sus orígenes históricos no era expresamente tan psicológica, ni egocéntrica y tenía más que ver con la moral, el honor, la reputación o el éxito. Así que también me voy a referir aquí a la parte popular y destinada al consumo en masa de ese complejo "psi" en términos de "complejo": el "complejo de autoayuda".

El complejo de la autoayuda se estructura en torno a unos pocos y reiterados temas que constituyen una estructura matriz de la "psicologización egótica" de la vida humana. El vocabulario de los títulos de los libros de autoayuda nos da una idea de esa matriz. Exceptuando libros con títulos efectistas o metafóricos (como La vaca o ¿Quién se ha llevado mi queso?, o algunos más "místicos" como El secreto o Manual del guerrero de la luz), los más descriptivos se ordenan en tres categorías: poder (atrévete, puedes, puedo, ser dueño, controlar, poder intuitivo, energía personal), cambio (aprender, me doy permiso, camino, reciclar, mejorar...) y estado personal final "pleno" (crecer, crecimiento personal, desarrollo personal, autorrealización, felicidad, paz interior, armonía, plenitud...). En síntesis, el poder cambiar para llegar a la meta de la autorrealización².

El esquema de la autoayuda es simple: (1) Victimización: la influencia externa convierte a la persona en un sujeto mutilado, apático, infeliz, sufriente, desgraciado...3. (2) Concienciación: esa misma persona puede cambiar la situación pero no lo sabe, por ende, necesita una mediación, una guía, una luz... (3) Empoderamiento: gracias a esa intervención la persona activa las fuerzas psíquicas internas necesarias para el cambio. (4) Resolución positiva de los problemas: el cambio tiene lugar.

La victimización conduce a la indefensión aprendida y a la negación de cualquier "poder" o "capacidad" para afrontar la vida. Sin el auxilio externo del consejero, del terapeuta o del grupo de autoayuda, el sujeto estará perdido. Se ha creado así el contexto previo a la intervención y es el sujeto victimizado (el yo-víctima: compulsivo, obseso, dependiente, perturbado, deficitario, no realizado, etc.) quien buscará activamente la ayuda externa. $Y$ encontrará sin 
esfuerzo y libremente una autoridad externa que le hará ver que ayuda desde la distancia asesorándolo y guiándolo sin constricciones ni imposiciones (Rose y Miller, 1992). La autoayuda se formula en un estilo cordial y cercano entre un lego y un experto, los dos interlocutores se ponen manos a la obra para crear una nueva referencia, una nueva realidad inmensurable (el yo y su pleno desarrollo) que, no obstante, es asible, cuantificable y controlable gracias a las exhaustivas y protocolarias "prácticas" y "técnicas" de autoconocimiento: actividades escritas, cuestionarios, ejercicios autoadministrados, pautas de comportamiento, inventarios, listas, escalas, sistemas de puntuación, etc. (Rimkie, 2000,68). De esas prácticas, se supone que surge el empoderamiento; supuestamente, el reconocimiento de las fuerzas psíquicas propias 0 , si se quiere, el endurecimiento caracterológico. Pero en realidad, funcionalmente el empoderamiento consiste en la creación de nuevas relaciones sociales, algo que queda oculto siempre en la retórica de la autoayuda. Y, sobre todo, el empoderamiento consiste en la modificación de las relaciones de podersaber entre expertos y legos: en las prácticas de autoayuda el experto (sea un individuo o un grupo, sea experto por especialización o por experiencia) comparte un saber psicoterapéutico con el lego y ambos se hacen coparticipes en la elaboración de nuevas definiciones de la realidad. No hay que menospreciar el valor terapéutico del "nuevo conocimiento". Ni la performatividad de los discursos del autoconocimiento: crean el mal que definen y también su curación. Pero el problema para aprehender este círculo vicioso consiste en que el mal es creado socialmente y la curación, sin embargo, se presenta individualizada en el formato de autoayuda: los individuos se responsabilizan de sí mismos y del cambio y control de sus vidas. Las nuevas condiciones interaccionales o incluso "sociológicas" se eluden sistemáticamente.

Efectivamente, el cambio no consiste en modificar o intentar modificar las condiciones socioculturales que han dado lugar al sufrimiento sino la propia "disposición psíquica" del sujeto: sus actitudes, valores, creencias, pensamientos, percepciones, etc. El núcleo clave de la autoayuda se relaciona directamente con lo que Kenneth Gergen (1996) denominó "discurso del déficit". Tal como ha señalado McGee (2005) a propósito de la autoayuda, las metas imposibles, mal definidas y huidizas (bienestar, realización, desarrollo, felicidad), convierten cualquier estado normal en un estado deficitario, incluso en enfermedad si el estado se prolon- ga. Como mucho se puede aceptar que la "situación de carencia" sea una etapa hacia la "plenitud" (las metáforas basadas en caminos, viajes y demás son recurrentes en todos los discursos de autoayuda). Y la vida entendida como camino está llena de lastres: de adicciones y dependencias que varan el desarrollo feliz de la persona. De ese modo, casi todos los aspectos de la vida humana se encuentran "patologizados" o necesitados de ayuda terapéutica. Las prácticas y las narraciones de autoayuda se extiende con éxito por doquier estableciendo "metas" imposibles ante las que todo estado "normal" acaba por ser anormal, deficitario y problemático.

Al aproximarnos al discurso y sus contenidos enseguida vemos cuál es el mundo problemático de la autoayuda, cuáles son los campos de la psicoterapia masiva: las relaciones interpersonales (el sexo, la pareja, el amor, la amistad.... $)^{4}$, los roles y su desempeño (la familia, el ser padres, los roles laborales...), y el autocontrol y el bienestar psíquico y corporal (el empleo, la salud, la felicidad...).

$Y$ en seguida percibimos cuál es el núcleo fuerte de esta psicoideología: querer es poder, y se debe querer. Si no sabes cómo querer déjate "autoayudar". Visto así parece que la autoayuda se instaura en la ruina del sujeto moderno que ha dejado de querer y que precisa de ayuda externa para poder recuperar el autocontrol e incluso para recuperar la motivación para el autocontrol. Lo que la autoayuda hace es dirigir las energías de cambio y de control a un territorio yermo: el de la psique desocializada. Los sujetos del complejo de la autoayuda encuentran en sus prácticas y en sus textos aquello que se les propone: ayuda. Pero es una ayuda envenenada porque funciona como sustituto (desocializante) de las estructuras sociales que hacen a las personas psicosocialmente competentes y psicológicamente proactivos.

\section{El COMPLEjo de La aUtOAyUdA Y el COMPLEJO "PSI": COMPETENCIAS Y TRANSFERENCIAS EN EL PARADIGMA EGOCÉNTRICO}

El complejo de la autoayuda es la cara "práctica" y "popular" de la Psicología entendida ésta como un paradigma epistémico o ideológico, es la psicología para y de las masas en el mundo del consumo (Starker 1989) y en la so- 
ciedad del conocimiento. En este sentido hay que apreciar el éxito de la autoayuda tal como hace Eva Illouz (2007, 2010). ¿Qué es lo que hace que sea una industria tan rentable, una cultura tan viva, un discurso tan penetrante, una práctica tan funcional?

La tesis foucaultiana de Nikolas Rose (1990, 1996; Miller y Rose, 1994) es que los gobiernos liberales avanzados gobiernan a distancia por medio de la "autonomía de los individuos" y con el concurso y mediación de los "psicoexpertos". Según Rose los "psico-expertos" proveen de tecnologias para la producción y regulación de individuos que son (o deben ser) autónomos y socialmente competentes. Sin embargo, ni autonomía ni competencia social son bienes ordinarios y precisamente el éxito del complejo de autoayuda nos indica que son bienes de consumo rápido: apreciados pero poco interiorizados. $Y$ ahí está la clave. Es decir, más que fijarnos obsesivamente en el discurso debemos prestar atención al uso de la autoayuda. Y cada una de las manifestaciones de la autoayuda tiene características de uso específicas. Por poner un ejemplo, los programas de televisión nutren de narraciones emocionales, expresiones y relatos que se repiten en la autoatribución de sentimientos y de emociones. Pero los libros de autoayuda con su formato "instructivo" en muy pocas ocasiones son seguidos al pie de la letra. Funcionan como los regímenes alimentarios, las tablas de ejercicios, etc. Son una guía referencial a la que se hace caso de manera relajada. Lo que la gente compra es, sobre todo, la ayuda "empaquetada" y el título, con la ilusión descreída de que leerá, confiará, cumplirá y solventará su problema. Algunos de los libros más exitosos son de esos que hay que tener por si acaso y de los que recomiendan amigos o familiares que han pasado por la misma situación. Es decir, son libros que funcionan como sustituto de la ayuda y la mutualidad social, pero, como siempre en la autoayuda, delegando en el sujeto la solución de su problema.

Otras son, sin embargo, las consecuencias de la extensión de términos, que no de conceptos, de las "psico-disciplinas". La relación entre disciplinas expertas y autoayuda es una relación simbiótica, se retroalimentan y expanden mutuamente. La autoayuda ha recogido y popularizado términos acuñados dentro de las disciplinas "psico-expertas" que a su vez habian reificado y naturalizado "entidades" y "estados" psicológicos. El movimiento es doble: los expertos figuran como autoridad que legitima las creencias básicas de la autoayuda y sus prácticas, pero la extensión en un terreno abonado por los cambios socioculturales, hace que la ciencia "psi" se perciba gracias a la autoayuda como un instrumento de avance, de progreso y de liberación. Incluso en las corrientes más críticas con las disciplinas científicas y con su rigor o su excesivo empirismo (corrientes espiritualistas, New Age y demás), no se pone en duda la existencia de una entidad psíquica individual (de un $Y o$, de un self, de un yo-mismo, etc.) que deba ser desarrollado, conducido a la plenitud o a la salud. Tampoco dudan, por supuesto, de la eficacia de las terapias centradas en el cambio personal.

Al analizar las consecuencias culturales del "discurso del déficit" Kenneth Gergen (1996) indicaba que a medida que los especialistas "tecnologizan, etiquetan y miden los problemas de la gente, los legos son descualificados en tanto que conocedores". Los expertos construyen discursos legítimos y los discursos legos son desautorizados. La gente, que normalmente acepta la expertise de los especialistas, es desautorizada para el uso de los términos de la psicoterapia. Pero, sin embargo, la difusión de la autoridad del "complejo psi" es también la difusión de sus saberes y conocimientos. De ese modo entramos en el círculo de mutua expansión que he mencionado: los especialistas refinan y sofistican sus instrumentos analíticos y terapéuticos y la gente los acepta modificándolos (simplificándolos, objetivándolos, etc.). Los lenguajes cotidianos se pueblan de curiosas sentencias pretendidamente "correctas", "neutrales", e incluso "científicas". El saber normativo es, por supuesto, el saber experto y se recurre a él, incluso cuando se supone que se critica o que se plantea algún tipo de alternativa a la psicoterapia "oficial", porque sus herramientas se han hecho herramientas del sentido común, de la doxa constituyente y constituida de la "realidad psíquica".

La escalada de sofisticación y extensión de la "mirada psicoterapéutica" multiplica las disfunciones o enfermedades psíquicas. En palabras de Kenneth Gergen: "Cuando términos como 'estrés' y 'agotamiento laboral' ingresan en el sentido común de la lengua vulgar, se convierten en lentes a través de las cuales cualquier profesional laboralmente activo puede examinar su vida y encontrarla defectuosa. Aquello que se valoraba como una 'ambición activa' puede ahora reconstruirse como 'adicción al trabajo', 'pulcramente vestido' puede redefinirse como 'narcisista' y el 'hombre autónomo' se convierte en alguien que se 'defiende de sus 
emociones"'. "Dad a la población los martillos del déficit mental, y el mundo social se llenará de clavos" (Gergen 1996, 200). Casi todo comportamiento, incluidos algunos funcional y normativamente corrientes, puede ser calificado de trastorno, complejo, manía o adicción. Lo que antes eran cuestiones morales o pertenecían al elenco de ritos e instituciones étnico-éticas se han psicologizado (el luto, por poner un ejemplo, ha pasado de ser un rito social a ser un reto del yo psicológico). La multiplicación de estos diagnósticos exige, como he dicho, la intervención masiva de expertos.

La autoayuda como corriente cultural se enraíza en la creencia en la determinación de la voluntad racional del yo, del poder intencional de la conciencia individual. La psicología y el psicoanálisis, sin embargo, pertenecen a las epistemologías de la sospecha: el ser humano está determinado por fuerzas que desconoce y que no puede por si solo dominar o controlar. La revolución científica y política consiste en desvelar la naturaleza de esas fuerzas y en dotar a los seres humanos del dominio de las mismas, de manera que puedan organizar su vida intencionalmente o, por lo menos, puedan ser conscientes de sus limitaciones.

La autoayuda se enmarca en principio en el modelo del yo activo, expresivo y volcado hacia fuera. Hasta su psicologización egocéntrica era parte del proyecto del self-mademan (era eminentemente masculina) y era una apuesta moral por el honor y la reputación (Illouz, 2010, 198). La psicología, por su lado, pertenecía de pleno al modelo del yo neurótico, su preocupación principal era desvelar las neurosis producto de la socialización y corregirlas (Drinka, 1984). Las psicologías del yo consiguieron traducir el lenguaje psicoterapéutico al discurso de la autoayuda y éste a su vez fue trasladándose desde lo moral a lo psicológico, al tiempo que las instituciones y las ideologías más "socializantes" se debilitaban (y lo políticopúblico se llenaba de lo político-privado y viceversa). De esas transferencias surgió el complejo de autoayuda tal como se conoce hoy en día. En el centro siguió imperando un modelo básico de psicoideología, el de la autodeterminación o autonomía personal, pero con una importante variación: la autodeterminación se psicologizó traduciendo la autonomía (sociopolítica y moral) en términos de potencialidad y desarrollo del yo. Términos que podian enmarcar narraciones biográficas pero al tomarse por requisitos de "salud" y "normalidad" acabaron produciendo la generalización del yo "enfermo", "carente" o "incompetente" (en una palabra del yo víctima). Y necesitado de ayuda (Furedi, 2003; Moskowitz, 2001).

La sociedad del conocimiento se caracteriza por la descarga de conocimiento experto en todos los ámbitos de la vida. Pero esas descargas no se dan en una única dirección, aunque los conocimientos legitimados sobredeterminan las traducciones, los intercambios y transferencias de conocimiento son multilaterales. También en la autoayuda: los ámbitos especializados, semiespecializados y legos son activamente productivos e innovadores y se contaminan e influye de manera que van borrando las fronteras que los delimitan. Eso obliga a que los expertos definen los instrumentos de su distinción y legitimación como autoridad. Pero el complejo "psi", desde su nacimiento, se ha volcado en la intervención directa y en la comunicación con sus "clientes" $y$, por ende, en la popularización como forma de legitimación de su expertise. No hace falta recurrir al Psicoanálisis donde es evidente que se "inviste" de conocimiento práctico, de un saber-hacer, al "paciente" (a pesar de considerar que es impotente sin el socorro del analista); Walden Dos, por ejemplo, no fue un libro de texto para terapeutas de la conducta. Y hoy en día es complicado decidir si los libros de Marie-France Hirigoyen, de Boris Cylrunik, de Mihayil Csikszentmihalyi o de Daniel Goleman son o no son libros de autoayuda. En realidad, ese carácter no reside en el texto, que puede estar más o menos cercano a las fórmulas retóricas de la autoayuda sino en el uso. Las transferencias son constates. Libros expertos acaban circulando en el mercado de best sellers de la autoayuda y libros de autoayuda son recogidos e integrados en la terapia experta ${ }^{5}$.

Estamos, por decirlo así, ante una alfabetización o evangelización general de las poblaciones del consumo en los códigos de interpretación y en las normas de comportamiento del complejo "psi". Esa evangelización "psíquica" precisa de formatos de consumo masivo, "breviarios" o "devocionarios", y de evangelizadores competentes en la comunicación "emocional y empática". Ha tenido escenarios privilegiados, predispuestos a funcionar como escuelas o misiones de la nueva fe. Algunos de esos escenarios son precisamente las instituciones que participaron en la primera modernización de las "conciencias": familia, escuela, empresa, ejército, hospital, etc. Ahora bien, la autoayuda no pertenece a ese 
primer modelo de modernización (disciplinante), sino a una oleada posterior enmarcada en las transformaciones de la Modernidad Avanzada o Hipermodernidad.

Durante la primera Modernidad se impulsó un modelo de Yo ideal en torno a las diferentes formas de autonomía: autonomía moral, cognitiva, emocional, etc. El rechazo a las diferencias sociales "heredadas" y "externas" se extendió junto con la creencia en un $Y_{0}$ interno, auténtico motor endógeno de la acción legítima y moral. La elaboración discursiva y práctica de ese $Y_{0}$ consistía en sustituir a los mediadores "externos" del comportamiento por mediadores "internos", es decir, que entre las condiciones del comportamiento y el comportamiento figurase el Yo y no un conjunto de disposiciones sociales: ritos, protocolos, etc. De esa manera, el Yo ha sido progresivamente "enclaustrado" en sí mismo y "cargado" de estructuras psíquicas individualizadas (habitus, actitudes, sentimientos, pensamientos, etc.).

La Hipermodernidad es la modernidad desinstitucionalizada: la vuelta del Yo del enclaustramiento a las redes de la socialidad natural podía haber devenido en su modelo ideal. Pero no ha sido así. La desinstitucionalización supone la pérdida de ciertos referentes superyoicos y el debilitamiento de ciertas mediaciones abstractas, estructurales o institucionales (del Otro Generalizado o de la Totalidad Social). En una época esto se consideró una liberación: el sujeto resurgía entre los fragmentos de los roles, la cultura subjetiva renacía entre las ruinas de la cultura objetiva. Desarrollo personal, autenticidad, personalización, humanización y otros términos quisieron dar cuenta de este reencuentro del Yo consigo mismo. Pero no ha habido tal encuentro. El Yo renacido y encumbrado al que ha dado lugar la hiposocialización ha sido y es un Yo narcisista que busca antes que nada encuentros fugaces consigo mismo en pantallas reflejas (un yo-imagen en un universo de relaciones en las que todos somos progresivamente más externos a los demás y más precarios).

Lo que normalmente se entiende como un proceso de psicologización y desocialización contrapone lo personal y lo social, continuando así con la presunción básica de la psicoideología del Yo abstracto, esto es, la oposición entre lo psicológico y lo sociológico. Esta falsa oposición no nos lleva a un yo más fuerte o más autocentrado, aun cuando exalta el Yo egocéntrico, va extendiendo nuevas formas de heteronomía "externalizadas" en las que se va sustituyendo lo social "directo" por mediaciones expertas y tecnológicas. Se sobreentiende que el complejo "psi" y el complejo de autoayuda han cumplido y cumplen un papel promotor en el desarrollo del proceso de concentración psicoegótica y de su actual externalización. Todo ello dentro de un vasto movimiento hacia la desconexión social, hacia la desvinculación de lazos, redes o círculos de relaciones inmediatas, espontáneas, sincrónicas y localizadas. Aunque siguen y es de suponer que seguirán existiendo, las relaciones no opcionales surgidas de los tiempos y los lugares comunes están sobredeterminadas por modelos de relaciones preseleccionadas, en las que la elaboración de comunidad está previamente acotada y altamente formalizada. La fragmentación de los vínculos sociales es general, pero su consumo opcional sigue siendo patrimonio de quienes poseen buen capital económico y cultural. Y lo mismo se puede decir de todos los dispositivos exopsíquicos presentados como servicios externos o bienes fungibles.

En definitiva, el complejo de autoayuda comercializa un modelo de Yo operativo y funcional en un mundo de densas interdependencias mediadas, de laxas instituciones "abiertas" y de consecuentes riesgos psicosociales. Faltan escenarios informales donde adquirir la competencia psicosocial necesaria para moverse en la inseguridad y la incertidumbre $y$, de momento, las generaciones que inauguran la Hipermodernidad siguen demandando algún sustituto funcional de los vínculos y la competencia psicosocial. No es extraño, por tanto, que en los modelos de autoayuda nos encontremos con un Yo ideal y con su correspondiente socialidad comunicativa que son más carencia que realidad. La realidad supuesta que legitima y hace funcional el discurso y las prácticas de la autoayuda es el cambio y el movimiento perpetuo: cambio social difícilmente controlable y predecible, combatido con cambio personal, proactividad e innovación. Veámoslo en uno de los ámbitos donde más claramente se ha producido el triunfo de las ingenierias psicosociales expertas y de los productos de psicoterapia lega: el manejo de las personas en el mundo laboral.

\section{El Nuevo Management y las demandas DEL CAPITALISMO FLEXIBLE}

Las transformaciones de la gestión o del gobierno de las empresas están intimamente relacionadas con las dife- 
rentes etapas del capitalismo y normalmente se describen como el paso desde sistemas de administración disciplinantes a tipos de gestión "más humanos" preocupados por la motivación y la satisfacción del trabajador (del taylorismo al humanismo). La última etapa de esta deriva se etiqueta de diversas maneras, aunque parece tener cierto consenso el rótulo Nuevo Management (Fairclough y Chiapello, 2002). Este nuevo tipo de gestión se corresponde con el éxito rotundo del complejo psi, es su versión en y para la gestión de las relaciones y las emociones en las organizaciones. Surgió con la intervención de los psicólogos en las empresas bajo el liderazgo de la figura de Elton Mayo que llamó la atención sobre el valor productivo de los sentimientos de los trabajadores. Con la influencia de Mayo las relaciones humanas y el estado emocional de los empleados empezó a considerarse como un elemento fundamental de la racionalidad económica bajo la luz de la racionalidad psicológica, es decir, el discurso experto sobre el manejo de individuos "disfuncionales" se empezó a aplicar en el mundo del trabajo; sobre todo alli donde el conflicto sociopolítico había sido previamente barrido por la supuestamente neutra intervención del Scientific Management de Taylor.

El Nuevo Management y su discurso siguen la estela del humanismo y en general de la psicologización del trabajo, de sus conflictos y sus problemas (Crespo et al., 2005; Fernández, 2007). Como tal se empezó a identificar en los años 80 y se puede considerar a In Search of Excellence de Peters y Waterman (1982) como el manifiesto fundacional del Nuevo Management. Alli, bajo el amparo de ideas fuertes como la excelencia o la calidad total se marcaban las grandes líneas de intervención: la consecución de productividad a través de los trabajadores convertidos en capital humano, la exigencia de autonomía e iniciativa al trabajador y la prioridad del cliente en todo el proceso productivo. En definitiva, la gente, sus relaciones, sus competencias y sus actitudes iban a ser el recurso fundamental de la era postindustrial.

Boltansky y Chiapello han defendido que el management nacido en los años 90 es propio del "tercer espíritu del capitalismo", de un capitalismo "conexionista" que ha integrado la crítica "artística" de los años 60/70 y se legitima en el imaginario de la "sociedad del conocimiento" (Boltansky y Chiapello, 1999). Estos nuevos discursos dan respuesta a las duras críticas que sufrieron las organizaciones burocráticas piramidales y sus formas de sujeción en los sesenta. Y han conseguido aplicar valores "revolucionarios" de entonces al mudo laboral y productivo: autonomía, creatividad, liderazgo, colectividad, equipos, comunidad, etc. Lo mismo viene a decir Richard Sennet (2006): los rebeldes de los sesenta se levantaron contra las grandes y rígidas organizaciones burocráticas, su derrumbe daría una oportunidad a la auténtica comunidad. Pero en lugar de esa comunidad mítica, lo que surgió fue el mercado con sus lógicas de poder sin regulaciones y de libre competencia que favorece a quien tiene de antemano una buena competencia, un buen capital. Y surgieron organizaciones "ligeras" y "en red" en las que R. Sennet (2006) observa tres déficit o carencias: la erosión de la fidelidad hacia la organización, la disminución de la confianza informal entre trabajadores (junto con las autoridades y liderazgo informales) y la pérdida del conocimiento o sabiduría organizacional.

Los procesos de desocialización generales se observan de cerca dentro de las empresas: las relaciones sociales informales se van perdiendo o bien se van haciendo cada vez más objeto de atención explícita por parte de expertos. El capital social que surgía de las interrelaciones de manera más o menos espontánea se ha convertido en un capital a producir, gestionar e invertir. Pero en la medida en que se carece de espacios y tiempos comunes, se confía en técnicas especializadas para producir sentimientos sociales (en numerosas ocasiones implementadas por consultores externos; de modo que los sentimientos sociales se adquieren como servicio). ¿Se puede comprar la adhesión de un trabajador? ¿La cohesión de un equipo? ¿Se pueden crear sentimientos y competencias sociales sin "círculos de relación" duraderos? El Nuevo Management dice que sí. Y para ello tiene a su disposición el complejo de autoayuda y las disciplinas "psi".

\subsection{La lógica del valor añadido: distinción, innovación y flexibilidad}

La lógica del valor añadido sintetiza los cambios que caracterizan al capitalismo global y flexible de las sociedades del consumo masivo y del conocimiento-comunicación. En las últimas décadas del siglo pasado el trabajo industrial perdió su hegemonía y un discurso "postproductivista" fue afianzando el nuevo capitalismo cognitivo. La acumulación cedió a la circulación y la producción de bienes materiales 
se subordinó a la producción de bienes inmateriales como modelo económico avanzado. Scott Lash resume todos esos cambios bajo el concepto de informacionalización (Lash, 2002). Lo que cuenta no es la materialidad del producto sino su forma, cuantificable y comercializable como información registrada, como propiedad intelectual. La pérdida de valor del producto erosiona la vieja diferencia entre valor de uso y valor de cambio y se impone el valor informacional que se produce tanto en el proceso productivo como en el uso del producto. Los bienes son sobre todo bienes simbólicos: no valen tanto por lo que son (materialidad), como por lo que significan (información). En el consumo y en la especulación global, la distinción es el valor por excelencia. $Y$ para ser distintivo y distinguido hay que investir al objeto de un valor intangible, un valor "añadido". No es sólo una labor de branding y marketing, el valor añadido se trabaja con esmero desde el principio de la producción de bienes y servicios. Ser competitivo en una economía informacionalizada significa ser distinto, creativo e innovador.

La innovación aparece junto con la creatividad y el cambio permanente como horizonte psicoeconómico básico. Cualquier agente económico debe ser móvil, adaptable y proactivo. Es decir, no vale con adaptarse a las demandas cambiantes del sistema, hay que tener la iniciativa de crear las propias condiciones en las que se jugará. La innovación es fundamentalmente esa proactividad que predefine $y$ predetermina el campo de juego. Las raíces de la proactividad como valor psicosocial se remontan al tiempo de las grandes programaciones pero tal como otros valores sociales y morales se ha convertido a un tiempo en una competencia psicológica, en un capital económico y en un recurso discursivo de legitimación. La meta-ideología del cambio político programado, que ha amparado a todas las ideologías, se ha traducido en la Hipermodernidad "postideológica" en términos de competencia psicosocial y de energía psíquica (un afán político movía al activista y al revolucionario, un afán creativo, casi artístico, mueve al innovador o al emprendedor).

El capitalismo global y flexible se inauguró con fuertes ajustes en los sistemas productivos, en las relaciones laborales y en las competencias personales. La desregulación general se presentó como el modo eficaz de impulsar la nueva libido capitalista: la del consumo de bienes simbólicos distintivos y la de la especulación con información.
La virtud correspondiente a ese mundo de consumo rápido, de vínculos precarios y superficiales y de inversión en información y conocimiento es la flexibilidad.

Desde un punto de vista psicosocial, la flexibilidad tiene dos aspectos a destacar: lo que está en juego dentro del mercado laboral es el valor añadido del trabajador desmaterializado (valen más sus competencias potenciales que su experiencia, su adaptabilidad que su adaptación, su predisposición que su posición, etc.) y, en consecuencia, las exigencias de la empleabilidad imponen un vaciamiento del psiquismo: cualquier disposición estable debe ser sacrificada a la adaptación rápida ${ }^{6}$. Paradójicamente la adaptación exige una alta competencia psicosocial (alta autoestima, autocontrol emocional, buenas habilidades en relaciones interpersonales o comunicativas, empatía y gestión emocional, etc.). Así que la adaptabilidad es al mismo tiempo carencia de disposiciones estables fuertemente incorporadas (de habitus) y predisposición a la innovación y al cambio en entornos volátiles. Esta última predisposición no es operativa si no está bien nutrida de apoyo externo y es ahí en donde funciona el complejo de autoayuda y las técnicas del Nuevo Management.

El trabajo inmaterial tiene dos caras: el trabajo intelectual o producción de conocimiento y gestión de información y el trabajo afectivo-asistencial (Hardt y Negri, 2004). En ambas caras impone unas competencias profesionales en las que la competencia "psicosocial" es central. En esas ocupaciones las relaciones y las emociones son objeto de trabajo, son el producto y son un aspecto principal del proceso. El estatus social de los trabajos de asistencia, cuidado y atención a las personas es por lo general bajo pero, sin embargo, las competencias especializadas en la comunicación, la interacción y la regulación emocional son altamente valoradas ${ }^{7}$. Y lo son porque crean valor añadido. La gestión eficaz del capital social da fortaleza a las empresas. Es ya un lugar común del nuevo capitalismo que las relaciones interpersonales y las disposiciones psíquicas (valores, actitudes, creencias, sentimientos, emociones, deseos, motivos...) son o pueden ser fuente de plusvalía (Henwood, 2003). Asi que no se deben descuidar la solidaridad, la afectividad, las relaciones, los estados emocionales, las expectativas, etc., de los trabajadores. Estos valores intangibles a veces se entienden como materia prima, pero por lo general el complejo psi los presenta como un producto altamente elaborado que no puede ser 
gestionado sin el concurso de psico-expertos. A no ser que los propios gestores se conviertan en psico-expertos.

\subsection{Vínculos precarios y manejo de las emociones}

Según Millward y Brewerton (2000) las relaciones entre trabajadores y empresa han pasado de un viejo pacto a uno nuevo. El viejo se establecía en torno a vínculos estables y a jerarquías reconocibles tanto en la recompensa como en el desempeño; en el viejo pacto el horizonte laboral se podía conjugar con el horizonte vital en una carrera personal y en una distribución de los ámbitos de relaciones (laboral, familiar, social...), todo ello en un contexto en el que las empresas creaban "sociedad" al establecer prácticas, identidades y referencias colectivas. Este modelo "socializante" ha sido sustituido por un nuevo pacto cuya característica central es la inestabilidad y la incertidumbre.

¿Cuándo se comenzó a hablar con tanto énfasis de satisfacción laboral, de implicación, de compromiso organizacional, de clima laboral, de cultura de empresa, etc.? Cuando con el nuevo pacto se fueron deshaciendo las vinculaciones psicosociales previas y se impuso la necesidad de crear explícitamente lo que antes se producía de manera no intencional. En la nueva cultura del capitalismo la empresa "ligera" ha acabado con las redes sociales informales, con la cultura informal, con el liderazgo psicosocial espontáneo o con el conocimiento colectivo (R. Sennet, 2000, 2006). No hay forma de organización que pueda subsistir sin esas dimensiones, así que cuando las condiciones formales no fomentan la vida informal de la organización se deben implementar técnicas para producir los efectos positivos de esa vida informal, pero sin ella.

Por poner un ejemplo, en los años sesenta Chris Argyris propuso el concepto de "contrato psicológico" en un libro titulado Understanding Organizational Behaviour. Según Argyris el contrato psicológico es un acuerdo implícito e informal acerca de las expectativas que mantienen empresa y trabajador sobre sus vinculaciones mutuas. Ese contrato surge, se mantiene y se modifica en las relaciones informales más que en las formales. Cuando es un buen contrato el trabajador está motivado, se implica e identifica con su labor e incluso muestra una adhesión psicológica con la empresa. La propuesta de Argyris, sin embargo, apenas tuvo eco hasta los años ochenta. Entonces con las oleadas de las primeras grandes desregulaciones, el ascen- so del neoliberalismo, la globalización de las grandes corporaciones y la exteriorización, se retomó el concepto con intereses prácticos: ¿Qué estaba pasando con todo eso que se establecía en el contrato psicológico? ¿Era y es posible establecer buenos y vinculantes contratos psicológicos de forma explícita a través de técnicas ad hoc y sin contratos laborales y formales estables? Evidentemente el contrato identitario de la empresa providencial iba desapareciendo y en su lugar políticas expresas pretendian producir los efectos de los vínculos anteriores: los efectos de los vínculos entre empresa y contexto social por medio de políticas de responsabilidad social corporativa y de "branding social" y los efectos de los vínculos entre empresa y trabajador por medio de las técnicas del Nuevo Management. Se podría así establecer un nuevo contrato psicológico meramente transaccional, a corto plazo y superficial, que no vincula más que para aspectos cubiertos por contrapartidas concretas (Roussseau y McLean, 1993).

Los encargados de esa misión, del restablecimiento de los vínculos psicológicos positivos, son los managers. Aunque cada vez más con el auxilio de consejeros, asesores y demás expertos externos, el peso de la gestión de los contratos psicológicos y sociales cae sobre las espaldas de directivos llamados a ser líderes de personas. Y es en ellos en quienes toma cuerpo el nuevo modelo de trabajador ideal, el trabajador competente del capitalismo flexible informacionalizado. Ahora bien, las nuevas estructuras en red, los equipos de proyectos, etc., hacen que casi cada trabajador se enfrente con el requisito de convertirse en un líder de si mismo y de su entorno laboral. Y en las respuestas que tenga hacia esa demanda estriba lo que hoy se conoce como "ciudadanía organizacional", altruismo organizacional, conducta organizacional prosocial, etc. Términos que vienen a decirnos que, sea cual sea la relación formal, los derechos y las obligaciones contractuales, la identificación con la empresa y su proyecto es el capital que demanda la organización, ahí radica el valor añadido del compromiso y de la motivación intrínseca, del emprendedurismo y del carácter innovador y creativo.

¿Cómo es un manager ideal? Y ¿cómo es, por ende, cualquier trabajador avocado a ser su propio "gestor"? No hay gran diferencia entre las descripciones que hacen los textos de autoayuda, los libros del Nuevo Management y los estudios "expertos". El modelo ideal nos describe a un trabajador con clara voluntad para el cambio y la 
adaptación, capacitado en el uso de nuevas tecnologías, con competencias lingüisticas y comunicacionales, con habilidades psicosociales en el manejo de las relaciones interpersonales y en su propio autocontrol emocional, con un pensamiento creativo y estratégico que le permite dominar situaciones críticas, solucionar problemas y "aprender a aprender". Es decir, las competencias psicosociales (comunicación, relaciones y automanejo) sustituyen casi por completo a las competencias profesionales clásicas. Y el trabajador (por supuesto, el líder) postfordista resulta ser un artista, un creador comprometido con su obra, su trabajo y su propia vida. Esto es lo que la autoayuda pretende: que cada cual sea un creador de su propia vida entendida como una obra de arte, el complemento necesario de un poder basado en la autoexplotación "creativa" de los individuos (McGee, 2005).

Eva Illouz ha descrito con acierto la introducción y el despliegue de toda esa narrativa terapéutica en las empresas $(2007,2010)$. De tal despliegue hay que destacar el papel que poco a poco fue adquiriendo la competencia emocional. Para Mayo el conflicto era el resultado de transacciones emocionales y se podía solventar con el reconocimiento de dichas emociones y la comprensión mutua mediante técnicas comunicacionales. Paradójicamente, la psicologización de los conflictos socioeconómicos o estructurales convertía a la empresa en el lugar donde se podían solucionar "disfunciones" y "trastornos" personales.

La intervención de Mayo no tuvo marcha atrás. Incluso cuando más tarde se introdujeron como legítimos los intereses personales o los conflictos interpersonales, el lenguaje de las relaciones y las emociones persistió (IIlouz 2010, 1000). La influencia de Carl Rogers, Abraham Maslow y de las teorías feministas reforzó la tendencia antiautoritaria y antidisciplinante dentro de las organizaciones, es decir, reforzó la necesidad de reformular las relaciones de poder de tal manera que lo que antes era choque físico directo y relaciones de control externo se convirtieran en manejo emocional y relaciones de control interiorizadas. Las expresiones emocionalmente contenidas empezaron a ser consideradas como estratégicas y la acción fundada en la expresión emocional desbocada y espontánea como algo disfuncional, hostil y conflictivo (la fuerza psicológica consiste en un dominio de sí mismo que asegure la consecución calculada de los intereses). En palabra de Eva Illouz: "... el tipo de control emocional requerido por los psicólogos combinaba dos atributos: la capacidad de ser racional en la búsqueda del interés propio y la capacidad para desactivar el conflicto y crear relaciones amigables" $(2010,109)$. Ese control se ha codificado como una competencia $y$, por tanto, como un capital (no es extraño, por tanto, que sean los directivos o managers quienes deban hacer mayores inversiones morales y emocionales para adquirir ese capital o en términos de competencias: para ser un buen líder). Ser emocionalmente competente se equipara a la expresión "suave" de las emociones de manera muy estandarizada o protocolizada y $\sin$ arrebatos espontáneos ${ }^{8}$.

La competencia emocional es, por tanto, una combinación de reflexión y escrutinio constante de las emociones propias para "neutralizar" su expresión junto con la observación inquisitiva de los posibles estados emocionales de los colaboradores o competidores. Este juego de indiferencias aparentes y calculadas indolencias es lo que Norbert Elias describió como "proceso de civilización". Lo que nos interesa señalar es que se ha convertido en el modelo ideal, normativo, adulto y saludable de las relaciones laborales y de las relaciones en general. La vida emocional-afectiva "privada" sigue la lógica de las relaciones y el intercambio económico (y la emoción se mercantiliza, se entiende como una transacción, Hochschild 2008) pero no tanto por la expansión economicista como por la expansión del discurso "psicoterapeútico" que impuso sobre las relaciones de solidaridad y vínculo socioafecticvo transacciones entre individuos psicológicos equivalentes y sustituyó las motivaciones morales engarzadas en las instituciones y roles colectivos por el interés particular y la biografía singular.

La gestión emocional se dirige, por un lado, a la estimulación (la motivación) y, por el otro, a evitar las confrontaciones interpersonales directas, abiertas y espontáneas, a evitar los conflictos organizacionales, sociopolíticos o de clase y a asegurar que todos esos problemas serán tratados terapéuticamente. En las organizaciones con recursos y bien gestionadas encontraremos ambiciosos planes de intervención emocional (con especial hincapié en la innovación), en los casos de profesionales preocupados por su empleabilidad la autoayuda sustituye al experto (especialmente como training en habilidades para el cambio "proactivo"). En uno y otro caso las lecturas críticas han denunciado la autoexplotación a la que nos conduce la gestión emocional. Pero hay numerosos casos en los que la gestión emocional se reduce a mera manipulación y 
herramienta de explotación externa: alli donde los vínculos formales no son más que transacciones precarias y frágiles en un universo de incertidumbres y desamparo institucional y social, la gestión emocional se presenta como un cínico instrumento de manipulación de conciencias?.

\subsection{Tópicos de autoayuda e innovación}

Las técnicas de control psicológico acompañadas de la pérdida de las relaciones sociales informales han hecho insoportable el conflicto y han conformado sujetos psicosocialmente incompetentes y emocionalmente apáticos. Sin embargo, la omnipresencia del cambio y de la incertidumbre en la ontología del discurso del Nuevo Capitalismo (Fairclough, 2000), exige un trabajo de adaptación constante del individuo que debe reinventarse en cada momento (McGee, 2005). El capitalismo de consumo y del valor añadido vive del cambio y de la innovación, necesita, por tanto, que los actores deseen cambios y sean capaces no sólo de consumirlos sino también de producirlos activamente. Buena parte de la intervención experta se dirige, por tanto, a estimular sujetos. A superar conformismos 0 barreras actitudinales contra la innovación y el cambio y a fomentar el deseo "normativo" de cambio.

Por otro lado, aun cuando el control emocional puede ser un buen capital individual para la integración organizacional, hace tiempo que algunas empresas se dieron cuenta de que era preferible recuperar la expresión de las emociones y comenzaron a reclutar sujetos "creativos e innovadores" de por sí, aunque fueran emocionalmente conflictivos. Ha surgido así un modelo de libido laboral siempre excitada que recupera figuras tradicionales del $Y_{0}$ proactivo: el conquistador, el aventurero, el pionero, etc. Figuras masculinas, por cierto. La energía psíquica que anima a estos "innovadores" es un capital escaso y apreciado por los gestores. Se invierte en estimulación externa y directa, es decir, en la creación de deseos e intereses personales sobrecargados discursivamente de referencias a la libertad, a la elección personal libre, a la posibilidad de plenitud o de desarrollo, a las potencias propias, etc. Una de las consecuencias de esta deriva proactiva e innovadora es la normativización de la motivación intrínseca y de la implicación personal. La motivación extrínseca, el cumplimiento de la tarea o la vinculación "transaccional" son disfuncionales y deben tratarse como patologías 0 potenciales patologías. Lo mismo ocurre con las relaciones interpersonales, todo lo que no sea una implicación activa en la creación de comunidad o equipo se entiende como desapego organizacional ${ }^{10}$.

En este panorama la autoayuda funciona como recurso de capitalización personal o como recurso para la motivación exógena, en ambos casos para adquirir el valor añadido de la vis innovadora, y en sus dos versiones tradicionales: la del método de autoayuda y la del grupo de autoayuda.

\section{El método de autoayuda para el cambio y la innovación: la} gestión de las emociones, de los marcos de percepción y de los conocimientos (es decir, de la carga cognitiva acumulada y de las destrezas, habilidades y competencias "situadas") sufren constantemente en ese horizonte sistémico donde el ciclo de vida de las competencias cognitivas, prácticas, emocionales y psicosociales es cada vez más corto. El yo progresivamente más efímero y más volátil debe encontrar sus apoyos en productos que pueda usar y tirar, productos de consumo rápido en los que se implica como usuario, preocupado únicamente por la relación calidad/precio y por los atractivos de diseño y branding del producto. Los dispositivos de autoayuda cumplen esa función: son complementos exopsíquicos. Liderazgo, personalidad, comunicación, creatividad, empatía, control emocional, iniciativa y otros temas parecidos se formulan de manera práctica y supuestamente operativa en guias y protocolos que descargan al sujeto del peso de esas propiedades psíquicas. Se trata de no acumular bienes inmuebles psíquicos y de sustituirlos por capital psíquico en continua circulación. La competencia psíquica moderna adquirida gracias a la incorporación de hábitos y de esfuerzo autodisciplinante, se sustituye por una competencia externa; podría decirse que el software psíquico moderno, instalado tras una costosa y larga descarga, es sustituido por software on line. En ese caso, sigue siendo fundamental tener lo que antes se consideraba una buena posición, hoy en día: una buena conexión. El habitus fundamental es el del consumidor ávido de novedades, deseoso de desear. Pero el habitus innovador no reside en el consumo (ni siquiera en el consumo de autoayuda), sino en la producción. Los sujetos innovadores son aquellos que todavía animados por deseos que van más allá del uso efímero de novedades, producen divergencias, nuevos escenarios, nuevos problemas y soluciones diferentes. Difícilmente encontraremos esa fuerza innovadora en los protocolos de la autoayuda. 
La organización como grupo de autoayuda: la intervención psico-experta pretende que la organización puede o debe ser una comunidad "tradicional" de autoayuda. Se hacen simulaciones de comunidad (dinámicas de grupo), se establecen culturas normativas comunes (formalizando de manera preestablecida lo que debería ser resultado de la práctica viva), se implementan sistemas y prácticas de comunicación "empática" sin participación de los implicados, etc. A veces funcionan, durante algún tiempo y para algunos miembros de la organización se restablecen los contratos psicológicos y se conforma un clima grupal positivo y fértil. Las demandas del sistema económico suelen acabar con esos logros efímeros. $Y$ vuelta a empezar. Pero en el camino también hay un desgaste psicosocial a tener en cuenta: cuanto mayor es la regulación de los expertos en los aspectos sociales informales de la organización, mayor es su desertificación. 0 , dicho de otro modo, mayor es el abandono de las relaciones sociales en el lugar del trabajo. El efecto es por tanto una desocialización rampante retroalimentada por esa intervención experta que pretende recomponer vínculos sociales, comunitarios y psicológicos. La cultura del complejo psi considera necesario ese motor de energías productivas que es el bienestar psicosocial, por eso los psico-expertos trabajan con ahínco en la intensificación de las relaciones sociales "consciente" y "formalmente elaboradas". Aunque el objetivo suele ser extender la competencia emocional y comunicacional e integrar a los trabajadores en grupos funcionales con buen clima, con ello se consigue retroalimentar el ciclo de la interpasividad y de la desconexión emocional. En definitiva, se hace aumentar la falta de estimulación o motivación endógena y se refuerza la dependencia de estimulación externa, una dependencia generalizada en la sociedad del consumo, del espectáculo y de la dependencia donde la apatía se combate a base de fuerte estimulación externa (publicitaría, propagandistica, evangelista o pornográfica).

Los aspectos mencionados (la zozobra del yo y la comunidad preestablecida) son complementarios y se sustentan mutuamente. El grupo que se presenta como generador de comunicación para el cambio, para la innovación o para la creatividad productiva o relacional, no está orientado al cambio de las reglas de juego (ni para repensarlas de forma crítica), ni está dirigido a entender las relaciones de influencia entre los numerosos círculos de relación en la que vive la empresa y sus trabajadores. Su labor es crear jugadores competentes (sean individuos, sean grupos). Y es ahí y para esos efectos donde se despliega el complejo de la autoayuda en las organizaciones.

Los dos puntos anteriores nos sitúan ante el marco en el que la innovación se vive como problema, o mejor dicho en el que la conversión de todos en agente innovador se convierte en un callejón sin salida: la falta de motivación se retroalimenta gracias al reduccionismo psicologizante y a las técnicas explícitas de motivación (Sprenger, 2005). La crisis de energía psíquica endógena se sostiene sobre un "malentendido": lo que se ha considerado endógeno y autónomo no era en realidad más que el producto condensado en los individuos ("tomado por individual") de energías sociales y de movimientos y motivaciones psicosociales, es decir, relacionales e institucionales. No es correcto, aunque sí muy coherente con la autoayuda, cargar con la culpa de la "crisis de motivación" a los individuos. $Y$ es esa forma de pensar y de organizar la que causa desmotivación y anula la influencia motivacional de las relaciones y de las instituciones.

La conciencia creciente del valor y la importancia de las dimensiones sociales de la persona y las relaciones sociales en todos los procesos innovadores, creativos y productivos de la vida (incluida la producción económica) es algo positivo. Puede, sin embargo, concretarse de diferentes maneras, en sintesis encontraremos dos tendencias: por un lado, tenemos la opción por el control formal de "unidades productivas individualizadas" con el auxilio de técnicas y dispositivos destinados a remendar sus carencias psicosociales $y$, por otro, la disposición de contextos, entornos o escenarios de conducta que den lugar a relaciones informales y conflictivas. La gestión de esta segunda opción exige liderazgo político capaz de crear comunidad alli donde hay antagonismo, es decir, capaz de crear "empresa" donde hay de hecho personas y grupos enfrentados según sus intereses y sus motivos sin negar la existencia de los mismos y sin menospreciar las vinculaciones formales. La primera opción suele transcurrir por vías menos políticas: parte de consensos inexistentes o apáticos, implementa medidas formales de intermediación e inhabilita la "creatividad disensual" de los individuos a los que, sin embargo, exige motivación endógena. No hay que decir que esta opción es psicosocialmente estéril para la innovación cognitiva y social. La autoayuda se encuentra ahí en su casa. 
Las demandas de la "lógica del valor añadido" propias del capitalismo de consumo conducen la innovación a una situación paradójica: por un lado, exigen innovación constante pero, al mismo tiempo, socavan las condiciones de la misma. Socavan en general cualquier forma de trabajo y vida que suponga implicación, esfuerzo, experimentación, aprendizaje, larga duración, atención exhaustiva, retrocorrección y retroalimentación, comunicación y socialidad densa e intensa. El complejo psi y sobre todo sus manifestaciones populares prescriben modelos de autorrealización y automotivación para la innovación endógena "en el aire", es decir, modelos de una innovación que supuestamente surgen del individuo como si fuera una potencialidad "psi- cológica" actualizada y convertida en competencia (y capital) por la pura fuerza de la voluntad personal. Se arranca al individuo de las relaciones constitutivas y se lo abstrae del entorno psicosocial. Se podria, no obstante, escapar a ese callejón sin salida, a esa innovación de consumo personal efímero, estableciendo modelos socioculturales de innovación. Eso exigiría inversión estratégica, diseños sociológicos e intervenciones políticas que creen socialidad $y$, desde el punto de vista psicosocial, sería preciso que el desarrollo de competencias, habilidades y hábitos se pegase al terreno, se hiciera en espacios y tiempos comunes y duraderos, creando círculos y redes de innovadores codependientes.

\section{NOTAS}

1 Trabajo realizado en el marco del proyecto de investigación Filosofía de las Tecnociencia Sociales FFI 200803599/FISO, financiado por el Ministerio de Ciencias e Innovación.

2 Son muchos los autores que han analizado el estilo retórico de la autoayuda, además de los trabajos generales sobre autoayuda de Salerno, Rimkie, McGee, etc., véase: Askehave (2004), Cowlishaw (2001), Cheng y College (2008), Lichterman (1992).

3 La victimización fue extendida según Salerno $(2005,28)$ por los grupos de ayuda mutua o de recuperación/rehabilitación (recovery groups), en especial por los Alcohólicos Anónimos y sus "doce pasos". Según Wendy Kaminer (1992) el alcoholismo fue presentado como una enfermedad fatalista y se extendieron con ello patrones de indefensión y debilitamiento personal.

4 La patologización de las relaciones y los conflictos interpersonales merece una mención especial. Se ha llegado a conformar una "rama" de la autoayuda para atender esas patologías bautizadas como "code- pendencia". Aunque en un principio se aludia a las situaciones conflictivas por las que pasaban aquellas personas que convivían con "adictos" o "dependientes", pronto se incluyó en la codependencia a las relaciones "obsesivas" e incluso al "apego". Por supuesto, la persona codependiente es una "víctima" indefensa que no es capaz de "gobernar su vida". La codependencia es el negativo del "desorden narcisista", la conversión en disfunción de ciertas virtudes místico-espirituales (el "darse a los demás") o incluso sociales (la asistencia solidaria). Como ejemplo se puede consultar la página web de Codependientes Anónimos (Coda): http://despertarcodependencia.com.

5 Es el caso de la biblioterapia: una práctica muy extendida que integra, junto con otros textos, materiales de autoayuda como apoyo a la terapia. Las tasas de uso por parte de psicoterapeutas de la biblioterapia y de los libros de autoayuda son altas y se han publicado algunos estudios que confirmarian su utilidad. Existen guías "autorizadas" de recursos de autoayuda (Norcross et al., 2000 y 2003; Anderson et al., 2005). Para
Recibido: 21 de junio de 2010

Aceptado: 27 de octubre de 2010 
una aproximación más crítica, ver Halliday, 1991.

6 Ésa es la tesis de Richard Sennet en La corrosión del carácter (2000). El carácter, definido como un conjunto de rasgos personales más o menos permanentes y reconocidos por los demás, habría sido corroído por la desestructuración y flexibilización de las carreras laborales que funcionaron durante una época como soporte psicosocial fundamental. Y hoy no hay tal soporte. El sujeto desprovisto de programación navega a la deriva: carente de criterios estructurados pierde la motivación para elaborar cursos de acción largos y continuados y se deja llevar apática e indolentemente, solo estallidos de hueca expresividad recuerdan lo que fue el sueño de un sujeto socializado para la automotivación duradera.

7 Así como cuidar y atender personas directamente tienen poca consideración, el guiar, liderar, organizar y manejar gente (en una palabra cualquier "ingeniería social" o "psicosocial") tiene muy buena consideración. La comunicación afectiva que se ocupa del cuidado y la comunicación efectiva que se ocupa del manejo, han sido atributos de mujeres y hombres respectivamente, esto parece marcar aun hoy los diferentes valores de ambos estilos comunicacionales (Russell Hochschild, 2008).

8 "La ética terapéutica del control de sí se presenta como un vasto intento cultural por inocular en los actores un modo de jugar al juego sin ser aparentemente movidos por él. Su objetivo es inocular una actitud indiferente, una actitud de no ser tomado por el juego, con el objetivo de asegurar los intereses propios" (Illouz, 2010, 137).
9 Casos de intervención masiva de psico-expertos como los de France Telecom o Foxconn Technology muestran con claridad hasta que punto la gestión emocional "psicopatologiza" los conflictos sociolaborales.

10 Esos innovadores dotados de energía propia, de una libido "creativa endógena", son atrapados por su propia pulsión en las "redes superyoicas" de la empresa que así explota el narcisismo creativo por medio del propio sujeto. En palabras de Žižek (2007, 90): "... la intolerable paradoja de esta 'desalienación postmoderna': ninguna tensión opone mis pulsiones creativas idiosincrásicas más íntimas a una Institución que no las aprecia y que desea reprimirlas para 'normalizarme': lo que pretende conseguir la conminación superyoica de la empresa postmoderna tipo Microsoft es, precisamente mi idiosincrásica creatividad". Una vez extinguida la vis ludica del sujeto, deja de ser útil para esa autoexplotación narcisista. Este modelo de innovador que disfruta explotando su propio deseo en el "marco de oportunidades" de una organización o incluso aventurándose por su propia cuenta, está sobredeterminando las representaciones del sujeto innovador.

\section{BIBLIOGRAFÍA}

Alonso, Luis Enrique (2002): Trabajo y posmodernidad. El empleo débil, Madrid: Fundamentos.

Anderson, L. et al. (2005): "Self-help books for depression: how can practitioners and patients make the right choice", en British Journal of General Practice, 55: 387-392.

Archer, Francois (2009): Diario de un hipermoderno, Madrid: Alianza.
Argyris, Chris (1960): Understanding organisational behaviour, Illinois: Dorsey Press.

Askehave, Inger (2004): "Book If Life is a Game - These are the Rules If Language is a Game - These are the Rules: A Search into the Rhetoric of the Spiritual Self-Help", en Discourse Society, 15: 5.

Boltansky, Luc y Chiapello, Eve (1999): Le Nouvel Esprit du Capitalisme, Paris: Gallimard.

Cheng, M. y College, R. (2008): "The selves of self-help books: Framing, argument, and audience construction for social and autonomous selves", en $L O R E, 6,2$ de mayo.

Cowlishaw, B. R. (2001): "Subjects are from Mars, objects are from Venus: constructions of the self in selfhelp", en Journal of Popular Culture, 35 (1).

Crespo, Eduardo; Revilla, J. C. y Serrano, A. (2005): "La psicologización política del trabajo", en José Romay et al. (eds), Psicología social y problemas sociales, 2, Madrid: Biblioteca Nueva.

Dolby, S. K. (2005): Self-Help Books: Why Americans Keep Reading Them, University of Illinois Press.

Drinka, G. F. (1984): The birth of neurosis: Myth, malady, and the Victorians, New York: Simon \& Schuster.

Fairelough, N. y Chiappello, E. (2002): "Understanding the New Management Ideology: A Transdisciplinary Contribution from Critical Discourse Analysis and New Sociology of Capitalism", en Discourse \& Society, 13(2): 185-208.

Fairelough, N. (2000): "Representaciones del cambio en el discurso neoliberal", en Cuaderno de Relaciones Laborales, 16: 13-35.

Fernández, C. J. (2007), El discurso del management, Madrid: Centro de Investigaciones Sociológicas. 
Furedi, F. (2003): Therapy Culture: Cultivating Vulnerability in an Uncertain Age, London: Routledge.

Gergen, K. J. (1996): "Las consecuencias culturales del discurso del déficit", en K. J. Gergen, Realidades y relaciones. Aproximaciones a la construcción social, Barcelona: Paidós.

Halliday, G. (1991): "Psychological self-help books: How dangerous are they?", en Psychotherapy: Theory, Research, Practice, Training, 28: 678-680.

Henwood, D. (2003): After the New Economy, New York: New Press.

Herman, E. (1995): The Romance of American Psychology. Political Culture in the Age of Experts, Berkeley: University of California Press.

Illouz, E. (2007): Intimidades congeladas. Las emociones en el capitalismo, Madrid: Katz.

Illouz, E. (2010): La salvación del alma moderna. Terapia, emociones y la cultura de la autoayuda, Madrid: Kazt.

Ingebly, D. (1985): "Professionals as socializers: the 'psy complex'", en Research in Law, Deviance and Social Control, 7: 79-109.

Lash, S. (2002): Critique of Information, London: Sage.

Lichertman, P. (1992): "Self-help reading as thin culture", en Media, Culture and Society, vol. 14: 421-447.
McGee, M. (2005): Self-Help, Inc. Makeover culture in American Life, New York: Oxford Univ. Press.

Miller, P. y Rose, Nikolas (1994): "On therapeutic authority: psychoanalytic expertise under advanced liberalism", en History of the Human Sciences, 7(30): 29-64.

Millward, L. J. y Brewerton, P. (2000): "Psychological contracts: Employee Relations for the Twenty-First Century", en C. L. Cooper y I. T. Robertson (eds.), International Review of Industrial and Organizational Psychology, 15: 1-60.

Moskowitz, E. S. (2001): In Therapy We Trust: America's Obsessions with SelfFulfilment, Baltimore: John Hopkins Univ. Press.

Norcross, J. C. et al. (2003): Authoritative guide to self-help resources in mental health, New York: Guilford Press.

Rieff, P. (1966): The Triumph of the Therapeutic: Uses of Faith after Freud, New York: Harper and Row.

Rimkie, H. M. (2000): "Governing citizens through self-help literature", en Cultural Studies, 14(1): 61-78.

Rose, N. y Miller, P. (1992), "Political power beyond the state: problematics of government", en British Journal of Sociology, 43(2): 173-205.

Rose, N. (1985): The Psycgology Complex, London: Routledge.
Rose, N. (1990): Governing the Soul: The Shaping of the Private Self, London: Routledge.

Rose, N. (1996): Inventing Ourselves: Psychology, Power and Personhood, Cambridge: CUP.

Rousseau, D. y McLean P. J. (1993): "The contracts of individuals and organizations", en L. L. Cummings y B. Stow (eds.), Research in organizational behavior, vol. 15, 1-47, Greenwich, CT: JAI Press.

Russel Hoschild, A. (2008): La mercantilización de la vida intima. Apuntes de la casa y el trabajo, Madrid: Katz.

Salerno, S. (2005): SHAM -Self-Help and Actualisation Movement: How the Gurus of the Self-Help Movement Make us Helpless, London-Boston: Nicholas Brealey Publishing.

Sennet, R. (2000): La corrosión del carácter. Las consecuencias personales del trabajo en el nuevo capitalismo, Barcelona: Anagrama.

Sennet, R. (2006): La cultura del nuevo capitalismo, Barcelona: Anagrama.

Starker, S. (1989): Oracle at the Supermarket: The American Preoccupation with Self-Help Books, New Brunswick: Transaction.

Zizek, S. (2007): En defensa de la intolerancia, Madrid: Sequitur. 